\title{
MOTIVATION FOR INDEPENDENT TRAVEL BY CHINESE TOURIST: A CASE STUDY OF FEMALE COLLEGE STUDENTS TRAVELLING TO SOUTHEAST ASIA
}

\author{
Qihang Qiu ${ }^{1}$, Tianxiang Zheng ${ }^{2}$
}

\begin{abstract}
Researches on recognition and classification of tourist motivation are of great significance to marketing and destination promotion. By utilizing push and pull theory as well as taking independent travelling to Southeast Asia as an example, 22 variables which affect the motivation of female college students were first identified. Then, factor analysis and descriptive statistics were conducted on the dataset to obtain five push factors including knowledge enrichment, relaxation and escape, sightseeing, cultural training and emotional communication, associated with four pull factors including convenience and trust, comfortable facilities and services, experience for better living and high-quality recreational activities. Findings also reveal that the top five factors (with respect to mean value) are all from push factors. Finally, some suggestions and improvement on tourism products in Southeast Asia are included accordingly.
\end{abstract}

Keywords - Independent Travel, Tourist Motivation, Female College Student

\section{INTRODUCTION}

As a new tour type, independent travel is unique and idiosyncratic due to its benefits from the convenience of modern civilization and its freedom from business patterns. Western scholars have been studying tourist behavior since 1930s[1]. They analyzed the behavioral characteristics of tourists from the perspective of sociology. Other studies focused on the classification of tourist motivations, with the intention of combining motivations with social demographic characteristics [2-4]. On the other hand, relevant researches in China started in the late 1980s, when Bao Jigang[5-8] discussed some topics on demands and motivations. Because tourism motivations are complicated and dynamic, methods of tourist motivation should be further developed in respond to different groups, destination and situation[9].

Previous study pointed out that modern tourists have the trend of developing from generalization to customization, from dependent requirement to individual independence [10]. Nowadays, the traditional mode of travelling could not satisfy an individual's need, and independent travel gradually dominates the mainstream of tourism market[11]. Considering $65 \%$ annual contribution to the world tourism industry is dominated by women[12], this study utilizes push and pull theory to investigate motivation for independent travel by Chinese female college students travelling to Southeast Asia. Clarifying this can improve the tourism industrial chains intensively, which is beneficial to developing marketing strategies on destination attractions, shops and other entertainment facilities.

The rest of the paper is organized as follows. Proposed research method is explained in section II. Experimental results are presented in section III. Concluding remarks are given in section IV.

\section{RESEARCH METHODOLOGY}

This study adopted the method of questionnaire survey for data collection to analyze the tourist motivations of female students travelling to Southeast Asia. Before conducting formal questionnaire, we read several relevant papers for references and developed a module which satisfies the psychological characteristics of female college students. Then we identified 22 possible variables influencing tourist motivation by making a pretest with 10 female students. After

${ }^{1}$ Shenzhen Tourism College, Jinan University, Guangdong 518053, China

${ }^{2}$ NU-UF International Joint Laboratory on Information Technology \& Tourism, Guangdong 518053, China 
that, we carried out the questionnaires which will be described in the following paragraph. Finally, we used factor analysis and descriptive statistics method with the help of IBM SPSS Statistics 19 to classify the motivation and analyze the obtained results.

The questionnaires were designated based on the previous similar researches with some modifications characterizing the independent travel motivation to Southeast Asia. We categorized motivation variables into two parts. The first part includes 22 questions about the tourist motivation, which consists of 12 push variables and 10 pull variables. The second part is relative to the respondents' personal information, where they were required to choose their genders, education levels, whether have been to Southeast Asia and the monthly consumption in order to exclude invalid questionnaire. A total of 230 questionnaires were hang out both online and offline, with 204 valid questionnaires returned. Likert 5 scale was used to measure the impact of these factors on tourist motivations (the value represents the recognition, i.e., $1=$ strongly disagree; $2=$ disagree; $3=$ do not know; $4=$ agree; $5=$ strongly agree).

\section{FINDINGS}

\section{A. Push Factors}

We used Cronbach to check the reliability coefficient of the push variables of questionnaire, where the Cronbach Alpha is 0.776 and the $\mathrm{KMO}$ value is 0.714; the Bartlett 's test value is 604.90 and it is significant under the conditions as the degree of freedom is 66 and the level is 0.000 , which indicates that there are some common push factors in the correlation matrix of the tourist motivation of female college students and the data is suitable for factor analysis.

In order to increase the significance of the research, the selection of each push variable is greater than 0.6, and finally there are 12 push variables involved in the analysis. The initial factor analysis was obtained according to the principal component extraction method, and then the orthogonal rotation method with maximum variance was used to rotate the extracted common factors. In line with the standard of larger-than-one characteristic root, a total of 5 factors were extracted, which explained $70.093 \%$ of the variance. Those five factors are: knowledge enrichment, relaxation and escape, sightseeing, cultural training and emotional communication, as shown in Table 1.

Table -1 Experiment Result for push factors

\begin{tabular}{|l|l|l|l|l|}
\hline Component & Sum Total & Variance(\%) & Accumulation(\%) & Name of factor \\
\hline 1 & 1.837 & 15.311 & 15.311 & Knowledge enrichment \\
\hline 2 & 1.814 & 15.12 & 30.43 & Relaxation and escape \\
\hline 3 & 1.657 & 13.881 & 44.242 & Sightseeing \\
\hline 4 & 1.574 & 13.119 & 57.361 & Cultural training \\
\hline 5 & 1.528 & 12.733 & 70.093 & Emotional communication \\
\hline
\end{tabular}

Note: method of extraction: principal component method of rotation: orthogonal rotation method with Kaiser standardized. Rotation appears convergence after 6 iterations

The first push factor includes three variables: " to see new things and broaden mental horizons (0.828)", "to experience different culture and ways of life (0.719) and "to share with others after travelling $(0.715)$ ", so it is named "knowledge enrichment" factor.

The second push factor includes three variables: "to escape from daily life and change surroundings temporarily $(0.829) "$ ", "to reward myself because of heavy work pressure (0.755)" and "to escape from loneliness (0.790)", therefore it is named "relaxation and escape" factor.

The third push factor includes two variables: "to appreciate and get close to beautiful natural scenery (0.837)" and "to visit the historical and cultural attractions $(0.811)$ ", so it is named "sightseeing" factor.

The fourth push factor includes two push variables: "to fulfill own interests and hobbies (photographing, painting and so on) (0.779)" and "to do academic research (0.774)", so it is named "cultural training" factor.

The fifth push factor includes two variables: "to enhance the relationship between friends or between family members $(0.804)$ " and "to experience by myself because a lot of friends and families have been to (0.765) ", so it is named "emotional communication" factor.

\section{B. Pull Factors}

We used Cronbach to check the reliability coefficient of 10 pull variables of questionnaire, where the Cronbach Alpha is 0.847 and the $\mathrm{KMO}$ value is 0.840 , which means that those 10 pull variables are suitable and this questionnaire is reliable; the Bartlett 's test value is 761.20 and it is significant under the conditions as the degree of freedom is 45 and 
the level is 0.000 , which indicates that there are some common pull factors in the correlation matrix of the tourist motivation of female college students and the data is suitable for factor analysis.

Again in order to increase the significance of the research, the selection of each pull variable is greater than 0.6, and finally there are 10 pull factors involved in the analysis. In order to make the result clearer, we selected 4 common pull factors with the standard of the characteristic root larger than 0.65 , which explained $70.049 \%$ of the variance. Those four factors are: convenience and trust, comfortable facilities and services, experience for better living and high-quality recreational activities, as shown in Table 2.

Table -2 Experiment Result for pull factors

\begin{tabular}{|l|l|l|l|l|}
\hline Component & Sum Total & Variance(\%) & Accumulation(\%) & Name of factor \\
\hline 1 & 1.765 & 19.268 & 19.268 & Convenience and trust \\
\hline 2 & 1.743 & 18.448 & 37.716 & Comfortable facilities and services \\
\hline 3 & 1.679 & 16.397 & 54.113 & Experience for a better living \\
\hline 4 & 1.624 & 15.936 & 70.049 & High-quality recreational activities \\
\hline
\end{tabular}

Note: method of extraction: principal component method of rotation: orthogonal rotation method with Kaiser standardized. Rotation appears convergence after 6 iterations

The first pull factor includes four variables: "short journey distance (0.842)", "attractive airfares (0.765), "low local price level and attractive product (0.763)" and "inspired by other people's experience (0.697)", so it is named "convenience and trust" factor.

The second pull factor includes two variables: "good social security conditions (0.875)" and "complete service facilities and lots of experiential activities (0.729)", therefore it is named "Comfortable facilities and services" factor.

The third pull factor includes two variables: "attractive food $(0.818)$ " and "friendly local citizens $(0.631)$ ", so it is named "experience for a better living" factor.

The fourth pull factor includes two variables: "can take part in diving, riding elephants and other recreational amusements $(0.831)$ " and "can enjoy tourism celebration and other holidays $(0.820)$ ", so it is named "High-quality recreational activities" factor.

\section{Top Contributive Factors}

After making descriptive statistics, we find that among these 204 copies of the data, five motivation variables have highest mean: "to see new things and broaden mental horizons (4.56)", "to experience different culture and ways of life (4.50), "to appreciate and get close to beautiful natural scenery (4.48)", "to share with others after travelling (4.26)" and "to visit famous historical and cultural attractions (4.23)", as shown in Table 3. These five variables all come from two push factors: "knowledge enrichment" and "sightseeing". The fact that the standard deviation of these variables are less than one means they are stable.

Table -3 Experiment Result for descriptive statistics

\begin{tabular}{|l|l|l|l|}
\hline Attributes & Mean & Standard deviation & Name of factor \\
\hline To see new things and broaden mental horizons & 4.56 & $\mathbf{0 . 6 4 4}$ & Knowledge enrichment \\
\hline To experience different culture and ways of life & 4.5 & $\mathbf{0 . 7 0 5}$ & Knowledge enrichment \\
\hline To appreciate and get close to beautiful natural scenery & 4.48 & $\mathbf{0 . 6 7 6}$ & Sightseeing \\
\hline To share with others after travelling & 4.26 & $\mathbf{0 . 7 6 1}$ & Knowledge enrichment \\
\hline To visit famous historical and cultural attractions & 4.23 & $\mathbf{0 . 8 1 7}$ & Sightseeing \\
\hline
\end{tabular}

\section{IV.CONCLUSION}

This paper investigates tourist motivation from two aspects: the external and intrinsic, and the factor analysis results show that the motivation for independent travel of female college students can be divided into five push factors including knowledge enrichment, relaxation and escape, sightseeing, cultural training and emotional communication, associated with four pull factors including convenience and trust, comfortable facilities and services, experience for better living and high-quality recreational activities. The findings also characterize the diversity of female students' motivations by identifying five top variables which have highest mean and contribute to two push factors namely "knowledge enrichment" and "sightseeing".

From the point of view of the push factors, enjoying new things and different experiences is the main reason that stimulate female college students to travel to Southeast Asia, which is in accordance with their gender and age. Besides, "relaxation and escape" is also an important motivation to female college students, for the heavy academic 
pressure and psychological needs. From the point of view of pull factors, economic expense of Southeast Asia tour is the leading reason, which means that female students will consider more about travel distance and cost when choosing a destination. From the point of view of findings on motivations, both motivation variables and the common factors show that the reason that encourages female students to travel stems much more from social psychological needs than the attractiveness of destination itself. In other words, the power of push is far greater than pull factors, and the fact that the five leading factors with highest mean derive all from female college students' social and psychological needs gives strong evidence to support this.

Last but not least, as to product development, because the motivations of female college students are different and dominated mainly by push factors, the owner or operator of scenic spots should pay more attention to facilities and recreational amusements to cater for this idiosyncratic market. Further, shops in such destination should increase the variety of products to satisfy the students' pursuit of novelty; the traffic departments should develop products and services with lower prices, considering the relatively low income of college students, for example, airlines may offer economical set meals to this type of passengers. As to product marketing, because the social and psychological motivations to female students are more appealing than the attractiveness of Southeast Asia, marketer of tourism products in Southeast Asia should concentrate on the products itself, so as to understand the core competitiveness of what idea you want to transfer to females as well as how and to what extent they will propagate your products.

To sum up, the scenic spots of a destination will benefit from the implication of our study, for instance, by tailoring advertising strategies so that female college students can be greatly attracted by Southeast Asia's "relaxation and escape", "knowledge enrichment" and other push factors. All these will help motivate them to travel to Southeast Asia.

\section{ACKNOWLEDGMENTS}

This work is supported by the National Natural Science Foundation of China (No. 41201145); Special Funds of Highlevel University Construction Program of Guangdong Province (Nos. 88015201 and 88016401); Jinan University Innovation Fund (No. 21617416).

\section{REFERENCES}

[1] Dann, G.M.S. Tourist Motivation: an Appraisal. Annals of Tourism Research 1981, 9, 187-219.

[2] Hritz, N.M.; Sidman, C.L.; D'Abundo, M. Segmenting the College Educated Generation Y Health and Wellness Traveler. Journal Of Travel \& Tourism Marketing 2014, 31, 132-145.

[3] Kim, S.S.; Prideaux, B. Marketing Implications Arising from a Comparative Study of International Pleasure Tourist Motivations and Other Travel-related Characteristics of Visitors to Korea. Tourism Management 2005, 26, 347-357.

[4] Ward, A. Segmenting the Senior Tourism Market in Ireland Based on Travel Motivations. Journal of Vacation Marketing 2014, 20, 267277.

[5] Bao, J. Study on Tourists 'Behaviors. Social Scientist 1987, 19-22.

[6] Wu, B.; Tang, J.; Huang, A.; Zhao, R.; Qiu, F.; Fang, F. A Study on Destination Choice Behavior of Chinese Urban Residents. Acta Geographica Sinica 1997, 52, 97-103.

[7] Lu, L. A Study on Motivations of Tourists in Mountain Resorts-- A Case Study of Tourists in Huang Shan Mountain. Human Geography $1997,12,6-10$.

[8] Liu, C. Research on Tourism Behavior and Motivation. Social Scientist 1992, 60-64.

[9] Zhang, H.; Lu, L. Summary on the Study of Tourist Motivation Abroad in the Recent Ten Years. Areal Research and Development 2005, 24, 60-64.

[10] Qin, M. Tourism Psychology. Peking University Press: Beijing, 2005; p 110-111.

[11] Huang, X.; Liu, C. Companion Constraints on Tourist Behavior: A Case Study of HongKong Ocean Park. Human Geography 2016, 31, 128-135.

[12] Analysis on Catchphrases. China Collective Economy 2010. 